\title{
Simposio de Endocrinología Obstétrica y Ginecología - Número 4 \\ Causas endocrinas de la esterilidad en la mujer: Diagnóstico y Tratamiento *
}

\author{
Dr. Edwin C. Hamblen**
}

(Traducción del Dr. G. E. P.)

Trataremos de resumir brevemente el tema el cual, en mi opinión, puede ser relativamente de poca importancia, pues, después de todo, hemos estado hablando durante todo el curso acerca de la deficiencia ovárica como tema fundamental, y la esterilidad de causa endocrina no es en realidad otra cosa que la deficiencia ovárica.

Hemos hablado ya de todos los tipos de deficiencia ovárica, las hemos clasificado desde el comienzo del curso, hemos tratado sobre hemorragia funcional, diferentes tipos de amenorrea, el tratamiento de todas estas anormalidades, y al hacerlo, no hemos hecho otra cosa que referirnos fundamentalmente a la esterilidad endocrina. Sin embargo, hay algunos puntos especiales que creo que merecen discutirse un poco más ampliamente.

En primer término, no debemos asumir que la esterilidad es de origen endocrino en lo que se refiere a una mujer, hasta tanto que la pareja haya sido sometida a un completo estudio. Igualmente, no debemos iniciar ningún tratamiento endocrino, específico o empírico, sin antes hacer la completa investigación del matrimonio. Esto es aplicable aún al tratamiento del llamado sín-

* Conferencid dictada por el autor en la Facultad de Medicina. - Universidad Javeriana. Bogotá, D. E.

** Profesor de Endocrinología de la Universidad de Duke Durhan N. C. Estados Unidos de América. 
drome de Stein Leventhal por medio de la resección cuneiforme de los ovarios. La única justificación que en mi concepto tiene la resección cuneiforme en los casos de Stein Leventhal, sería que permitiría a la mujer lograr un embarazo, y si ella no desea un hijo, quizás no haya razón en entusiasmarse con este método de tratamiento del síndrome. Si la paciente va a ser sometida a la resección cuneiforme de ovarios con el objeto de lograr un embarazo, ciertamente todo procedimiento quirúrgico debe estar precedido de una completa investigación de ella y de su esposo para estar seguros de que todos los demás factores son normales y que no habrá nada que le impida concebir si se puede corregir la deficiencia ovárica en cuestión. Por estas razones pienso y repito que, antes de decidir que estamos frente a un problema de esterilidad endocrina, debemos investigar completamente tanto a la mujer como al esposo.

Por supuesto, no creo necesario describir ante Uds. cómo se investiga el matrimonio estéril; Uds. lo saben suficientemente, y no es precisamente el tema de esta conferencia. Recuerdo muy bien cuando, hace unos 20 años, tuvimos por primera vez a la disposición la gonadotropina equina. Se pensó entonces que era la solución definitiva de todos los problemas de esterilidad endocrina, y algunos médicos, antes de tomar la historia clínica de la pa. ciente, daban de una vez inyecciones de gonadotropina equina. Nu es ciertamente esta la manera de afrontar el problema de la esterilidad.

Muchas veces encontramos que para afrontar correctamente el problema hay que tener largas conversaciones con la pareja estéril, para explicarle el por qué de todos los procedimientos de estudios que van a practicarse, su razón de ser, y lo que se debe esperar de ellos. Para obviar estas dificultades publiqué hace unos años un librito ("Facts for childless couples") para ser distribuído a las parejas estériles, y que nos ha ayudado mucho en la explicación de todos los detalles de su estudio, y quizás también a que comprendan por qué a veces tardamos tanto tiempo para poder decirles cuál creemos que sea la causa de su infertilidad, lo mismo que explicarles que si se encuentra una determinada causa, qué es lo que se puede hacer y qué lo que se debe esperar. Este librito sigue todavía siendo muy popular y es usado por muchos médicos de los Estados Unidos para instruír a sus pacientes. Incidentalmente, el niño cuya fotografía ilustra la portada, 
es el primer niño que pude obtener con la terapéutica gonadotrópica cíclica, y que ya hoy en día tiene un grado universitario. $\mathrm{Su}$ madre pudo lograr dos hijos con tres ciclos de tratamiento, así: en eil primer ciclo tuvo este niño, en el segundo no se logró embarazo, y en el tercero obtuvo su segundo hijo, lo cual considero muy interesante desde el punto de vista histórico.

Cuando hablamos de esterilidad endocrina, creo que debemos tratar de llegar a estadísticas críticas y reales. En nuestra Universidad tenemos actualmente unos 1.000 matrimonios que han sido adecuadamente investigados y tratados, y que considero un grupo que puede ya darnos datos estadísticos dignos de confianza. Tomo mis cifras de los primeros 400 por lo que fueron casos que investigué personalmente, pero puedo asegurar a Uds. que los 600 restantes dan datos sensiblemente iguales a los que voy a presentar hoy.

Pero surge enseguida un importante problema cuando hablamos de esterilidad endocrina. Me parece que para poder llegar a tener una concepción adecuada del problema debemos tomar solamente a las mujeres de parejas que han venido a nosotros para averiguar la razón por la cual no han podido tener hijos, y para solicitar el tratamiento correspondiente en caso de que fuere practicable. Hago especial énfasis en este punto, porque cualquiera puede tomar como casos de esterilidad endocrina todos los problemas de deficiencia ovárica que encuentre y ponerlos dentro de la estadística, lo cual aumentaría considerablemente las cifras de incidencia de esterilidad endocrina, ya que en una institución como nuestra Universidad, en que vemos tantas niñas que no tuvieron una pubertad normal, tantos casos de amenorrea, tantas mujeres con ciclos anovulatorios que en realidad no pueden considerarse como casos de esterilidad porque no tuvieron el problema, si tomamos todos estos casos como base para la estadística de esterilidad endocrina, no creo que tengamos una representación fiel de lo que entendemos cuando el Sr. y la Sra. X vienen a nosotros para saber por qué no tienen hijos, y a buscar solución a su problema.

Las cifras estadísticas en relación con esterilidad endocrina en la mujer son muy variables, y hay quienes dan cifras hasta del $35 \%$ de esterilidad de causa endocrina sobre el total de los datos de esterilidad, cifra que me parece muy alta. Por ejemplo, la más alta incidencia que tenemos nosotros de ningún factor es- 
pecial, es la oclusión tubaria bilateral que forma el $50 \%$ de nuestros casos, cifra que también me parece inusitadamente alta. Pero es que nosotros diagnosticamos la oclusión tubaria sobre la base de individualizar este factor y apreciarlo debidamente, o sobre la base del hecho de que quizá hubiera alguna obstrucción temporal de las trompas, que se resuelve en el curso de la primera insuflación. Por esto tenemos una cifra un poco alta de obstrucción tubaria.

Ahora, si contemplamos el problema de la esterilidad en la mujer, debida a causas puramente endocrinas, encontramos que en nuestras series es relativamente baja. Por ejemplo, en nuestras 400 parejas pudimos diagnosticar insuficiencia tiroidea en solo un $7 \%$, y solo $1 / 5$ de ese $7 \%$ tuvo deficiencia ovárica correlativa y diagnosticable. Estas cifras hacen realmente del factor tiroideo un factor muy bajo entre nosotros, que en otros países puede ser mayor. Además, esta estadística nos muestra una cosa que ya sabíamos; muchas mujeres con pequeño hipotiroidismo no son aún estériles, pueden concebir, pueden eventualmente tener abortos por el hipotiroidismo no tratado aún, o pueden concebir $y$ dar a luz niños hipotiroidianos.

Muchos autores confieren al factor corvical una importancia especialísima, hasta el punto en que asumimos que si el moco cervical no está suficientemente liquidificado, no se hace penetrable a los espermatozoides en el tiempo de la ovulación, pudiendo este factor ser considerado como causa endocrina de la infertilidad porque creen que la licuefacción del moco cervical alrededor del tiempo de la ovulación se debe al aumento del nivel estrogénico, y que se tiene un moco grueso, viscoso y no penetrable por los espermatozoides después de la ovulación, por la acción de la progesterona. Así, conocemos muchas personas entusiastas que cuando encuentran un moco cervical grueso y viscoso al tiempo con la ovulación, tratan a sus pacientes con pequeñas cantidades de estrógenos dadas antes de la ovulación, con el objeto de liquidificar el moco cervical. Por supuesto, si se dan dosis altas de estrógenos, se suspendería la ovulación. Mi punto de vista a este respecto es que si la paciente muestra plena evidencia de función ovárica normal, o sea que hay ovulación, no veo dónde está la razón de derle dosis pequeñas de estrógenos, porque creo que en esas circunstancias solo parece lógico relacionar la no preparación adecuada del moco cervical a una causa intrínseca del cervix, sea mal drenaje, 
sea endocervicitis, etc. Así, dudo que haya un factor endocrino que obre específicamente para disminuír la función del moco cervical, salvo en las mujeres en que hay un déficit ovárico notorio, y que por consiguiente les falta la ovulación, encontrándose entonces en otra parte la causa de la esterilidad.

En relación con el tratamiento, las cifras que presento podrían hacerne aparecer ante Uds. como un nihilista terapéutico o persona con un sentido demasiado crítico para interpretar los datos. Pero daré a Uds. mis opiniones personales, basadas en los resultados obtenidos con las 400 mujeres de los 400 matrimonios estudiados. Como figura global, ocurrieron embarazos en el 42,5\% de los matrimonios. Haciendo un análisis crítico de estos datos, encontramos solamente un $1 \%$ de casos de causa endocrina tratados con éxito, lo cual hace un contraste muy marcado con la cifra de $46 \%$ de embarazos referidos al tratamiento de la oclusión tubaria. En otras palabras, casi la mitad de los embarazos que pudimos conseguir, se refirieron a haber solucionado problemas de obstrucción tubaria. Y ahora, si analizamos la estadística referente a las 4 mujeres, de entre las 400 parejas que lograron embarazos con terapia endocrina, obtenemos lo siguiente: Una de las mujeres tuvo un embarazo que se pudo relacionar estrictamente con el tratamiento de un hipotiroidismo. Una tuvo un embarazo directamente relacionado con un estado de hipoprogestinismo y fué tratado por medio de estrógenos y progesterona suministrados después de la ovulación. Dos tuvieron embarazos correlacionados con terapia para déficit ovulatorio hecha con gonadotropinas equina y coriónica. Ciertamente, el factor endocrino, cuando se investiga siguiendo las normas que hemos indicado, no parece ser muy alto. Creo que la mayor dificultad para todos nosotros está en interpretar adecuadamente qué es lo que pasa con la terapia endocrina, pues ciertamente, si damos a una mujer un poco de tiroides por 3 ó 4 meses y queda embarazada un año después, no podemos decir que el embarazo se debió al tratamiento tiroideo.

Así, conozco a muchos de los que llamo "Endocrinólogos", que no vacilarían en hacer una tal correlación. Y creo también que es muy difícil disociar una respuesta específica al tratamiento, de la respuesta al impacto psicológico que Uds. tienen sobre sus pacientes, y en muchos casos la terapéutica psíquica se confunde con el tratamiento mismo. Por esta razón no veo y no pue- 
do entender cómo algunos presentan cifras tan altas de esterilidad endocrina, mucho mayores de las que aquí hemos presentado, y cómo pueden ser tan entusiastas de la terapéutica endocrina en esterilidad.

Terminaremos este resumen con algunos comentarios breves. No veo razón para resumir aquí el tratamiento del déficit ovulatorio pues ya se habló suficientemente de ello cuando se trató de amenorrea. No hablaremos de la resección cuneiforme de ovarios en el llamado síndrome de Stein Leventhal, pues ya lo hemos discutido in extenso. Lo mismo para los casos de fase luteal corta o hipoprogestinismo, el tratamiento de la hiperplasia adreno-cortical con cortisona o hidrocortisona, y además conductas que permitirían una reaparición de los ciclos ovulatorios. O el uso del tiroides en los hipotiroidismos, pues una mujer que tenga un hipotiroidismo con o sin déficit ovulatorio concomitante, debe recibir terapia tiroidea. Y como lo dijo antes, no voy a discutir la conducta de los que prescriben medicación tiroidea en el tratamiento de la deficiencia ovárica, de la hemorragia funcional, de la amenorrea o aún en el tratamiento empírico de mujeres que tienen una función ovárica normal, y en las que la terapia por tiroides podría ayudar. Sé que no es buena medicina académica, pero el punto importante es que a veces se obtienen buenos resultados. En fin, como otra vez lo dejé claramente establecido, no soy partidario de la irradiación de los ovarios o de la hipófisis con dosis bajas para tratar de lograr que las mujeres puedan concebir.

Por último, debo manifestar que muchos casos de esterilidad, sea que estén asociados con diversos grados de déficit ovárico, sea que no podamos establecer precisamente la causa, pueden tener relación directa con disturbios psicológicos (causa hipotalámica). Sabemos muy bien los buenos resultados que una paciente puede obtener sólo con entrar en contacto con un médico competente y comprensivo, y también todos conocemos los casos de parejas estériles que adoptan un niño, para comenzar inmediatamente a llenar su familia con hijos propios. 